\title{
UVC fluencies for preventative treatment of pseudomonas aeruginosa contaminated polymer tubes
}

Bak, Jimmy; Ladefoged, Søren D.; Begovic, Tanja; Winding, Anne

Published in:

Biofouling

Link to article, DOI:

$10.1080 / 08927014.2010 .520314$

Publication date:

2010

Document Version

Publisher's PDF, also known as Version of record

Link back to DTU Orbit

Citation (APA):

Bak, J., Ladefoged, S. D., Begovic, T., \& Winding, A. (2010). UVC fluencies for preventative treatment of pseudomonas aeruginosa contaminated polymer tubes. Biofouling, 26(7), 821 - 828.

https://doi.org/10.1080/08927014.2010.520314

\section{General rights}

Copyright and moral rights for the publications made accessible in the public portal are retained by the authors and/or other copyright owners and it is a condition of accessing publications that users recognise and abide by the legal requirements associated with these rights.

- Users may download and print one copy of any publication from the public portal for the purpose of private study or research.

- You may not further distribute the material or use it for any profit-making activity or commercial gain

- You may freely distribute the URL identifying the publication in the public portal 
This article was downloaded by: [DEFF]

On: 6 October 2010

Access details: Access Details: [subscription number 910491762]

Publisher Taylor \& Francis

Informa Ltd Registered in England and Wales Registered Number: 1072954 Registered office: Mortimer House, 3741 Mortimer Street, London W1T 3JH, UK

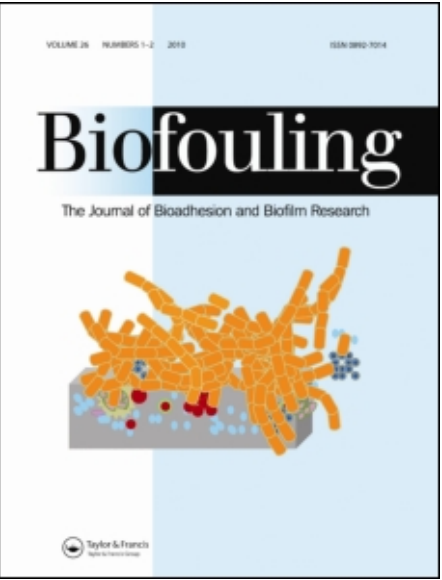

\section{Biofouling}

Publication details, including instructions for authors and subscription information:

http://www.informaworld.com/smpp/title content=t713454511

\section{UVC fluencies for preventative treatment of Pseudomonas aeruginosa contaminated polymer tubes}

Jimmy Bak'; Søren D. Ladefoged ${ }^{b}$; Tanja Begovic $c^{\mathrm{a}}$; Anne Winding ${ }^{\mathrm{c}}$

${ }^{a}$ DTU Fotonik, Roskilde, Denmark ${ }^{\mathrm{b}}$ Nephrological Clinic, Rigshospitalet, Copenhagen, Denmark ${ }^{\mathrm{c}}$

Department of Environmental Chemistry and Microbiology, NERI, Aarhus University, Roskilde,

Denmark

First published on: 20 September 2010

To cite this Article Bak, Jimmy, Ladefoged, Søren D. , Begovic, Tanja and Winding, Anne(2010) 'UVC fluencies for preventative treatment of Pseudomonas aeruginosa contaminated polymer tubes', Biofouling, 26: 7, 821 - 828, First published on: 20 September 2010 (iFirst)

To link to this Article: DOI: $10.1080 / 08927014.2010 .520314$

URL: http://dx.doi.org/10.1080/08927014.2010.520314

\section{PLEASE SCROLL DOWN FOR ARTICLE}

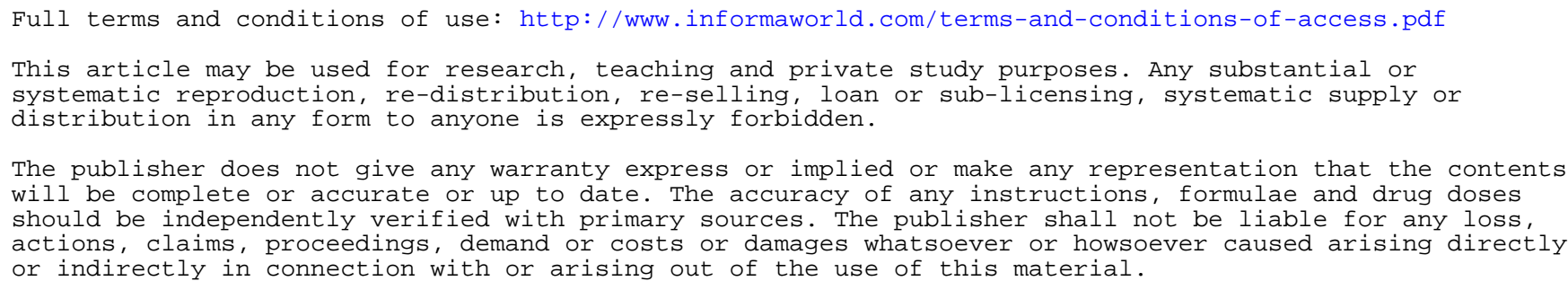




\title{
UVC fluencies for preventative treatment of Pseudomonas aeruginosa contaminated polymer tubes
}

\author{
Jimmy Bak ${ }^{\mathrm{a} *}$, Søren D. Ladefoged ${ }^{\mathrm{b}}$, Tanja Begovic ${ }^{\mathrm{a}}$ and Anne Winding ${ }^{\mathrm{c}}$ \\ ${ }^{a}$ DTU Fotonik, Frederiksborgvej 399, 4000, Roskilde, Denmark; ${ }^{b}$ Nephrological Clinic, Rigshospitalet, Copenhagen, Denmark; \\ ${ }^{c}$ Department of Environmental Chemistry and Microbiology, NERI, Aarhus University, Roskilde, Denmark
}

(Received 23 March 2010; final version received 26 August 2010)

\begin{abstract}
Exposing Pseudomonas aeruginosa biofilm grown on the inner surface of Teflon and silicone tubes to UVC light $(265 \mathrm{~nm})$ from light emitting diodes (LED) has previously been shown to substantially reduce biofilm growth. Smaller UVC fluencies were required to disinfect Teflon tubes compared to silicone tubes. Light propagation enhancement in tubes can be obtained if the refractive index of the intra-luminal saline solution is higher than that of the polymer. This condition is achieved by using Teflon tubes with a low refractive index (1.34) instead of the polymers with a high refractive index (1.40-1.50) normally used for tubing in catheter production. Determining whether or not UVC light exposure can disinfect and maintain the intra-luminal number of colony forming units (CFUs) at an exceedingly low level and thus avoid the growth and establishment of biofilm is of interest. The use of UVC diodes is demonstrated to be a preventative disinfection treatment on tubes made of Teflon, which enhances the UVC light propagation, and on tubes made of a softer material, ethylene vinyl acetate (EVA), which is suitable for catheters but much less suitable for UVC light propagation. Simulating an aseptic breach $\left(\sim 10^{3}-10^{4} \mathrm{CFU} \mathrm{ml} \mathrm{m}^{-1}\right)$, the UVC disinfection set-up was demonstrated using tubes contaminated with planktonic $P$. aeruginosa. After the tubes $(10-20 \mathrm{~cm})$ were inoculated with the bacterial solution for $3 \mathrm{~h}$, they were emptied and filled with saline solutions $(0.9-20 \%)$. Next UVC fluencies $\left(0-21 \mathrm{~mJ} \mathrm{~cm}^{-2}\right)$ were applied to the tubes $3 \mathrm{~h}$ after inoculation. Colony counts were carried out on liquid samples drawn from the tubes the first day after UVC treatment and liquid and surface samples were collected and analyzed 3-4 days later. A fluence of approximately $1.0 \mathrm{~mJ} \mathrm{~cm}{ }^{-2}$ was noted as being sufficient for no growth for a period of 3-4 days for the Teflon tubes. Determining the fluence threshold for the EVA tubes was not possible. Almost all of the UVC-treated EVA tubes were disinfected simply by filling the tubes with a saline solution. Direct UVC treatment of the contaminated EVA tubes revealed, however, that a fluence of $21 \mathrm{~mJ} \mathrm{~cm}{ }^{-2}$ killed the bacteria present in the tubes and kept them disinfected for a period of 3-4 days.
\end{abstract}

Keywords: biofilm; UVC; light emitting diodes; disinfection; sterilization; Pseudomonas aeruginosa; tubes; catheters; catheter sepsis; catheter bacteremia; catheter lumen; liquid light guide; refractive index; sodium chloride; fluoroethylene propylene; Teflon; ethylene vinyl acetate; UV transmittance in tubes

\section{Introduction}

Catheter-related infections caused by bacterial growth in long-term catheters are responsible for substantial morbidity and mortality, and impose a heavy burden on healthcare systems worldwide (O'Grady 2002; Donlan 2008). It is generally accepted that biofilms form shortly after catheter placement and that biofilm formation is the basis for catheter-related infection. Therefore, methods that can prevent or eradicate catheter biofilms are of substantial clinical interest.

UVC light emitting diodes (LED) were used recently for almost $100 \%$ disinfection of tube lumens contaminated with thin Pseudomonas aeruginosa biofilms (Bak et al. 2010). It was demonstrated that especially tubes made of fluoro ethylene propylene (FEP) Teflon were effectively disinfected with small
UVC fluencies (8-16 $\mathrm{mJ} \mathrm{cm}^{-2}$ ) corresponding to exposure times between 15 and $30 \mathrm{~min}$. Longer treatment times (up to $300 \mathrm{~min}$ ) were required to disinfect catheter tubes made of materials such as silicone (peritoneal catheter samples) corresponding to a fluence (exposure time) of approximately $170 \mathrm{~mJ}$ $\mathrm{cm}^{-2}$. This observation can be explained by the difference in the refractive indices between the various tube materials. FEP is known to have a much lower refractive index $(\sim 1.34$ at visible wavelengths) compared to other polymers such as polyurethane (PUR), silicone and ethylene vinyl acetate (EVA), which have refractive indices between 1.40 and 1.50. Tubes filled with a solution with a higher refractive index than that of the tube material itself were found to support intra-luminal UV light propagation efficiently by using the liquid itself as a light guide (Diemer et al. 1997). A relatively high

*Corresponding author. Email: jiba@fotonik.dtu.dk

Published online 27 September 2010 
refractive index (1.368) was obtained by preparing a $20 \% \mathrm{NaCl}$ solution. Using this type of solution made it possible to obtain $>50 \%$ UVC light transmittance in $10 \mathrm{~cm}$ tubes made of FEP.

Based on the documented efficiency of the UVC method against established $P$. aeruginosa biofilm (Bak et al. 2010), an examination was made of how small the UVC fluencies (exposure times) need to be to disinfect tubes infected accidentally by a clinically relevant number of CFUs. If the UV method is used preventively each time the catheter is accessed, formation of intraluminal biofilm should be reduced to a minimum. The numerous sources of catheter contamination include, for example, the patient, staff, external equipment and machinery (Donlan 2001; Raad and Hanna 2002; Menyhay and Maki 2006). Simulating a realistic aseptic breach during catheter handling, Shah et al. (2002) tested a taurolodine/citrate solution, which is a bactericidal agent, on moderately and newly contaminated catheters. The number of bacteria for this aseptic breach was in the range $50-500 \mathrm{CFU} \mathrm{ml}^{-1}$. Inspired by the work of Shah et al. (2002), results are presented here of a study using UVC LED for the disinfection of polymer tubes which have been exposed to an aseptic breach for a short period of time (a few hours).

As mentioned above Teflon tubes with a low refractive index have excellent UVC light guide properties when filled with a high refractive index solution. The mechanical properties of Teflon are, however, not optimal for producing central venous catheters because the material is rigid, making it difficult to mold and glue to other materials (Ash 2007). Softer polymers such as PUR and EVA are well suited for producing catheters, but do not have the same UVC light guide properties as Teflon because of their high refractive indices. As a result studies were carried out to determine whether or not it is possible to disinfect the aforementioned soft tubes, which can be used for central venous catheters with relatively low fluencies. The necessary fluencies to obtain no growth were also determined. Both a low refractive index polymer (eg FEP) and a soft polymer with a high refractive index (eg EVA) were inoculated and stored for a period of time with $P$. aeruginosa before UVC exposure was applied. The FEP tubes were included in order to compare the results (doses) obtained by the preventative approach to those observed earlier on the same tubes contaminated with biofilm.

The disinfection experiments presented here were designed to simulate a dialysis session. The aseptic breach was maintained for $3 \mathrm{~h}$ (duration of a typical dialysis session). Then the tubes were emptied and filled with saline solutions before they were UV treated. Next, liquid samples were drawn from the lumen and analyzed. The tubes were stored for an additional 3-4 days (typical time between two dialysis sessions) before liquid sampling and analysis were repeated and samples from the inner surface were also collected and analyzed.

\section{Materials and methods}

\section{Procedure for simulating an aseptic breach}

The following experimental procedure is valid for the samples prepared for later UVC treatment and for the parallel samples maintained as controls. Samples for UVC treatment and controls were stored under the same conditions during the experiments. A P. aeruginosa culture (clinical strain, local ID: 5322, isolated from a patient with urinary infection 2003) was used in every experiment. The culture was stored in serum broth, $+10 \%$ glycerol in $1 \mathrm{ml}$ vials at $-80^{\circ} \mathrm{C}$ and revived by spreading on Luria Broth (LB) agar plates. After a 24-h incubation, one or two colonies were selected from the plates and suspended in $5 \mathrm{ml}$ of $5 \%$ serum broth (Rigshospitalet, Copenhagen). The suspension was kept at $37^{\circ} \mathrm{C}$ for $2-3 \mathrm{~h}\left(\sim 10^{5} \mathrm{CFU} \mathrm{ml}^{-1}\right)$ before being suspended in $100 \mathrm{ml}$ of nutrient broth, (Herlev Hospital, Copenhagen). This suspension was further diluted in 11 of $0.9 \%$ saline solution (Fluka). After incubation overnight, $0.1 \mathrm{ml}$ of this solution was further diluted into $99.9 \mathrm{ml}$ of a sterile $0.9 \%$ saline solution. The number of microorganisms inoculated in the tubes was found to be in the range $10^{3}-10^{4} \mathrm{CFU}$ $\mathrm{ml}^{-1}$, which is somewhat higher than the level reported by Shah et al. (2002). After inoculation the tubes were maintained in a horizontal position for $3 \mathrm{~h}$ at $37^{\circ} \mathrm{C}$. Next, the tubes were emptied (without flushing) and filled with the various saline solutions before commencing the UVC treatment. The total volume of inoculums and replacement fluid was $1.3 \mathrm{ml}(10 \mathrm{~cm}$ tubes) and $2.5 \mathrm{ml}$ (20 cm tubes). After injection of the saline solution the tubes for UVC treatment were sealed with two windows made of UV-grade quartz (90\% transmittance) and mounted on an adjustable stage. During a normal dialysis session the catheter lumen is emptied of blood and flushed with a $0.9 \%$ saline solution before the lock solution (heparin) is injected into the lumen. In practice, the UVC disinfection could then be administered just before the lock solution (for instance heparin) is injected. The effect of the UVC treatment on the bacteria in the luminal liquid was analyzed immediately after UVC exposure and again after 3-4 days, at which time the bacteria on the inner surface were also counted. Control samples were stored under the same conditions as the UVC treated samples, ie with the same residence time, temperature and percentage of $\mathrm{NaCl}$. All incubations 
were at $37^{\circ} \mathrm{C}$. Figure 1 depicts the overall experimental procedure.

\section{Bacterial counting}

Only $0.1 \mathrm{ml}$ samples (total volume $2.5 \mathrm{ml}$ ) were drawn from the tubes on the first day in order to maintain the aseptic breach for a period of 3-4 days. Ten-fold serial dilutions of each sample were made in $0.9 \%$ saline solutions $(0.1 \mathrm{ml}$ from the first suspension to $0.9 \mathrm{ml}$ saline). Next, $100 \mu \mathrm{l}$ samples were spread on LB agar plates and incubated aerobically at $37^{\circ} \mathrm{C}$ for $24 \mathrm{~h}$. Direct plating of $0.1 \mathrm{ml}$ from the suspension gave a detection limit of $33 \mathrm{CFU} \mathrm{ml}{ }^{-1}$ (three replicates). After incubation, the number of CFUs was determined for the UVC-treated luminal solutions and controls. The number of CFU ml ${ }^{-1}$ was calculated if growth was observed. All plates were in triplicate from the same replicate to lower the detection limit. The tubes were then stored for an additional 3-4 days at $37^{\circ} \mathrm{C}$ (the period between two dialysis sessions) to study regrowth or effects of the high salt concentrations. This final analysis included both liquid sampling from the lumen analogous to the first day and the samples taken from the entire inner tube wall with a pipette brush in order to collect cells attached to the inner tube surface. Liquid samples $(0.1 \mathrm{ml})$ were drawn off the tubes and plated directly. The detection limit of the liquid samples on days 3 and 4 was $3.3 \mathrm{CFU} \mathrm{ml^{-1 }}$ (3 replicates). The pipette brush was used to remove attached cells from the inner tube wall from UVC treated tubes and the controls. The pipette brushes were sterilized overnight in $70 \%$ ethanol prior to use. The collected samples were flushed into a vial with $5 \mathrm{ml}$ of $5 \%$ serum broth and shaken in a vortex mixer for $30 \mathrm{~s}$ to disintegrate aggregated cells. Samples $(0.1 \mathrm{ml})$ were drawn from this suspension and plated directly. The detection limit was $3.3 \mathrm{CFU} \mathrm{m}^{-1}$ (3 replicates). This corresponds to $\sim 16 \mathrm{CFU}$ for

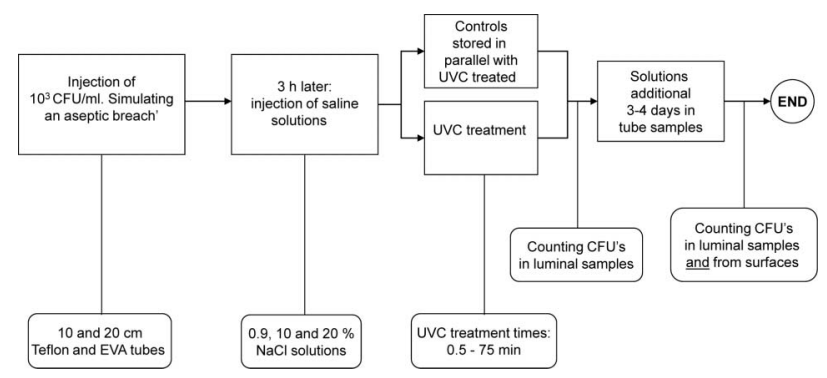

Figure 1. The overall experimental procedure used to test the efficiency of UVC exposure for disinfection of tubes in a 4 to 4 -day period. The test procedure was designed to simulate a dialysis session in which the catheter is accessed every third day. the entire $20 \mathrm{~cm}$ tube $\left(5 \mathrm{ml} \times 3.3 \mathrm{CFU} \mathrm{m}{ }^{-1}\right)$ and

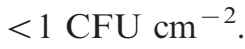

\section{UV disinfection set-up and test conditions}

A figure illustrating the UVC disinfection set-up can be found in Bak et al. (2010). The UVC light source was a $265 \mathrm{~nm}$ UVTOP LED from Sensor Electronic Technology Inc. Soldered to an electrical connector placed at one end, the diode was powered by a $6 \mathrm{~V}$ DC power supply. A ball lens placed on top of the LED focused the light into a small spot specified by the manufacturer to be $\sim 1.5-2 \mathrm{~mm}$ in diameter at a focal length of $15-20 \mathrm{~mm}$. The angle of the light cone launched into the tube openings was approximately $6^{\circ}$. The total output power from the continuous wave operated LEDs (electrical current: $I_{\mathrm{F}}=20 \mathrm{~mA}$ ) was measured with a UV detector (Blak-Ray, model J-225). The total UVC output $(\sim 0.25 \mathrm{~mW})$ was close to that specified by the manufacturer at $265 \mathrm{~nm}$ with the specified electrical current. The tubes were exposed to UVC light at intervals ranging from $30 \mathrm{~s}$ to $75 \mathrm{~min}$. The power emitted from the diode at $265 \mathrm{~nm}$ was 80-150 $\mu \mathrm{W}$. The UVC fluencies were $0.13-21.1 \mathrm{~mJ}$ $\mathrm{cm}^{-2}$. Tubes made of FEP Teflon and EVA were used in the disinfection tests. The lengths of the tubes were 10 and $20 \mathrm{~cm}$. The outer and inner diameter of both types of tube was 6 and $4 \mathrm{~mm}$, respectively. Pure $\mathrm{NaCl}$ (Fluka) was used to make the saline solutions $(0.9,10$ and $20 \% \mathrm{NaCl}$ ). The $20 \%$ saline solution was chosen in order to maximize the refractive index of the lumen liquid in the FEP tubes. This process showed that such a high concentration of saline solution is difficult to maintain in the tubes without precipitation of the $\mathrm{NaCl}$. The disinfection efficiency using $\mathrm{NaCl}$ solutions in concentrations $<20 \%$, for instance $10 \%$, for maintaining a good UVC light propagation should therefore be tested. According to the reference data a $10 \%$ solution corresponds to a refractive index $>1.35$, which is well above that of FEP (1.34). Nothing is gained with respect to UVC light guidance if a high percentage $\mathrm{NaCl}$ solution is used in tubes made of PUR and EVA. Tests were therefore carried out to determine whether it is possible to disinfect moderately contaminated tubes (both FEP and EVA) using a physiological saline solution $(0.9 \%)$. Disinfection of the soft EVA tubes filled with a $0.9 \%$ saline solution would be of clinical importance.

\section{Results \\ Test on FEP tubes}

Figure 2 shows the measured transmittances through $20 \mathrm{~cm}$ FEP Teflon tubes as a function of $\mathrm{NaCl}$ concentration. A refractive index higher than that of the 
FEP tube material $(n=1.34)$ can be obtained with $\mathrm{NaCl}$ solutions of $\sim 4-5 \%$. The transmittance in $20 \mathrm{~cm}$ FEP Teflon tubes was approximately $40 \%$ for $20 \%$ saline, $35 \%$ for $10 \%$ saline, $\sim 12-13 \%$ for a $0.9 \%$ saline solution and $10 \%$ for pure water. The refractive indices at visible wavelengths of saline solutions taken from the literature (Weast 19771978) are plotted on the same figure. The refractive index of FEP Teflon is reported to be 1.34 (Texloc 2005). The results of applying UVC exposure on $10 \mathrm{~cm}$

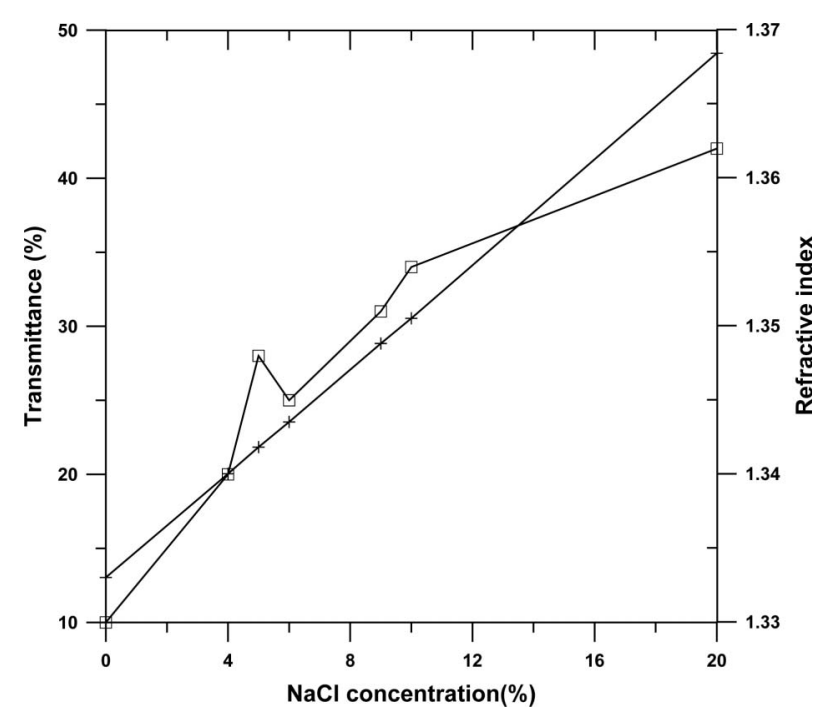

Figure 2. The measured UVC transmittance through $20 \mathrm{~cm}$ FEP Teflon tubes as a function of $\mathrm{NaCl}$ concentration $(\square)$. The refractive indices as a function of $\mathrm{NaCl}$ concentration from Weast 1977-1978 are also plotted $(\times)$. The transmittance through a $20 \mathrm{~cm}$ FEP tube filled with pure water is approximately $10 \%$. and $20 \mathrm{~cm}$ FEP tubes with a 10 and $20 \%$ saline solution are shown in Table 1. The FEP tube lengths were the same as those used in the authors' earlier work on biofilm. The disinfection results are made comparable by expressing the UVC fluencies exposed on the inner tube surfaces in $\mathrm{mJ} \mathrm{cm}{ }^{-2}$. No CFUs were observed on any of the UVC-treated liquid or surface samples. The control samples represent the aseptic breach and contain the original solution of $P$. aeruginosa injected into the tube lumens. The number of CFUs in the control samples was more or less constant at $10^{3}-10^{4} \mathrm{CFU} \mathrm{ml^{-1 }}$ during the observation time (3-4 days). The number of CFUs in the saline control solutions replacing the control samples is displayed in rows 3 and 4 (saline control). The number of bacteria in the saline control samples diminished rapidly from $\sim 10^{2}$ when analyzed on the first day to below detection limit. The reduction in the number of CFUs during the 3-4-day period could be caused by the high saline concentrations (10 and 20\%) and/or the absence of nutrients due to the dilution in sterile saline solutions. No plate counts were observed, even with the miniscule fluencies applied on the first day $\left(0.29\right.$ and $\left.0.58 \mathrm{~mJ} \mathrm{~cm}{ }^{-2}\right)$. Sufficient to maintain disinfection for a 3-day period in the lumen liquid and on the surface, these fluencies correspond to $30-60 \mathrm{~s}$ of treatment time.

UVC exposure was further applied to contaminated FEP tubes which were injected with $0.9 \%$ saline solutions that replaced the aseptic breach solution. In order to determine a threshold value for which almost $100 \%$ disinfection was obtained and re-growth was not observed, treatment times (fluencies) were augmented from very short time periods to those where no re-growth was observed on samples taken after 3-4

Table 1. Disinfection results on 10 and $20 \mathrm{~cm}$ FEP tube samples with $10 \%\left({ }^{\mathrm{a}}\right)$ and $20 \%\left({ }^{\mathrm{b}}\right)$ saline solutions.

\begin{tabular}{|c|c|c|c|c|}
\hline \multirow[b]{2}{*}{ Samples } & \multirow{2}{*}{$\begin{array}{l}\text { Mean fluence } \\
\mathrm{mJ} \mathrm{cm}^{-2}\end{array}$} & \multicolumn{3}{|c|}{ 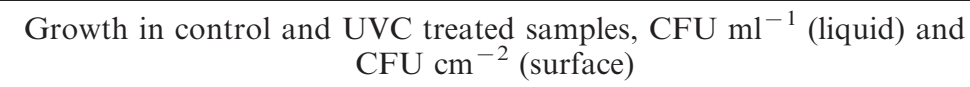 } \\
\hline & & Day 1 liquid & Day 3-4 liquid & Day 3-4 surface \\
\hline Control (4) ${ }^{\mathrm{a}}$ & - & $4.5 \times 10^{3}(1.3)$ & $7.8 \times 10^{4}(5.8)$ & $2.2 \times 10^{4}(1.6)$ \\
\hline Control $(3)^{\mathrm{b}}$ & & $4.0 \times 10^{3}(1.2)$ & $8.9 \times 10^{4}(6.3)$ & $1.5 \times 10^{4}(4.4)$ \\
\hline Saline control (3) ${ }^{\mathrm{a}}$ & - & $9.4 \times 10^{2}(11.0)$ & 3 & No growth \\
\hline Saline control (4) & & $2.0 \times 10^{2}(2.0)$ & No growth & No growth \\
\hline UVC treated $(1)^{\mathrm{a}}$ & 0.29 & No growth & No growth & No growth \\
\hline UVC treated $(1)^{\mathrm{b}}$ & 0.58 & No growth & No growth & No growth \\
\hline UVC treated $(1)^{\mathrm{a}}$ & 1.45 & No growth & No growth & No growth \\
\hline UVC treated $(2)^{a, b}$ & 2.91 & No growth & No growth & No growth \\
\hline UVC treated $(1)^{\mathrm{a}}$ & 5.80 & No growth & No growth & No growth \\
\hline UVC treated $(1)^{\mathrm{a}}$ & 8.71 & No growth & No growth & No growth \\
\hline UVC treated $(3)^{a, b}$ & 11.66 & No growth & No growth & No growth \\
\hline
\end{tabular}

The fluencies exposed to the inner tube surfaces are expressed in $\mathrm{mJ}$ per unit area. As the transmittance in 10 and $20 \% \mathrm{NaCl}$ is comparable $(35-40 \%)$, these treatments are combined. The range of treatment times varied from 1 to $30 \mathrm{~min}$. The detection limits are $33 \mathrm{CFU} \mathrm{ml}{ }^{-1}$ (day 1 ) and $3.3 \mathrm{CFU} \mathrm{ml}^{-1}$ (days 3 and 4). The numbers in parentheses in column 1 are the number of replicates. Average CFU counts and SDs (numbers in parentheses) of the controls are shown in the last three columns. 
days. Table 2 displays the disinfection results obtained on $20 \mathrm{~cm}$ FEP tubes filled with a $0.9 \%$ saline solution. The applied fluencies were between 0.13 and $5.62 \mathrm{~mJ}$ $\mathrm{cm}^{-2}(\sim 0.5-20 \mathrm{~min})$. Table 2 contains data from two sets of measurements separated by an interval of several months. The two sets of disinfection tests were run under nearly similar conditions. The only difference was that in the later experiment the tubes were discarded after each experiment and replaced with new ones. The level of contamination in the aseptic breach is comparable to the other tests on FEP tubes. The $0.9 \%$ saline solutions that replaced the aseptic breach solution after $3 \mathrm{~h}$ maintained a varying level of contamination during the 3-4 days of observation time. There was no growth in any of the UVC-treated samples on day 1 . Re-growth was only observed after $3-4$ days if the fluence was very low $(\sim 0.13-0.27 \mathrm{~mJ}$ $\mathrm{cm}^{-2}$ ). No re-growth was observed when the fluence was $>1.0 \mathrm{~mJ} \mathrm{~cm}{ }^{-2}$. This fluence is probably close to the threshold that kept the $20 \mathrm{~cm}$ FEP tubes filled with $0.9 \%$ saline solution disinfected for a 3-4-day period. The relatively high number of CFUs in the $0.9 \%$ saline control samples compared to none being observed in the 10 and 20\% saline controls (Tables 1 and 2) indicates that the high saline concentration kills the planktonic bacteria present in the tube lumens.

\section{Test on EVA tubes}

The aseptic breach model and UVC disinfection procedure were also applied to EVA tubes following the same scheme as for the FEP Teflon tubes. The literature shows that this polymer has a high refractive index of 1.45 (TexLoc 2005) relative to saline solutions. Earlier findings showed that the UVC light transmittance in PUR and silicone tubes was very poor (Bak et al. 2010). Therefore, nothing is gained with respect to transmittance by filling these tubes with a concentrated saline solution. Consequently, only $0.9 \%$ saline solutions have been used as the light propagation medium prior to UVC treatment. Table 3 shows the results of applying UVC exposure to $20 \mathrm{~cm}$ EVA tubes. The contamination level of the control samples is observed to be comparable to that observed for the FEP tubes for a 3-4-day period $\left(\sim 10^{4} \mathrm{CFU} \mathrm{ml}^{-1}\right.$ and $\mathrm{cm}^{-2}$ ). Table 3 also indicates that the initial level in the $0.9 \%$ saline control samples was lower on day 1 and apparently diminished substantially during the 3-4-day observation period towards no growth. On day 1, no CFUs were visible on the plates representing liquid samples from the UVC treated tubes. The UVC treated samples gave no growth with applied fluencies $>8.7 \mathrm{~mJ} \mathrm{~cm}{ }^{-2}$. It is difficult, however, to determine from the results in Table 3 whether or not the UVC treatment is the major cause of low CFU counts in the EVA tubes filled with $0.9 \%$ saline solutions as the contamination level in the $0.9 \%$ saline control samples was substantially lower than that observed for the FEP tubes (see Table 2). Additional tests in which the UVC exposure was applied directly to the $P$. aeruginosa solution (same contamination as the control) were therefore carried out. The results of these tests are shown in Table 4. The contamination level in the control samples was comparable to that observed earlier $\left(\sim 10^{4} \mathrm{CFU} \mathrm{ml} \mathrm{m}^{-1}\right.$ and $\left.\mathrm{cm}^{-2}\right)$. In this case, the EVA tubes were exposed to a higher fluence to demonstrate that direct UVC disinfection of these tubes was achievable. Table 4 shows that one plate also had CFUs, ie the 3-4-day liquid $\left(2.2 \times 10^{2} \mathrm{CFU}\right.$ $\mathrm{ml}^{-1}$ ). In conclusion, all liquid samples taken from

Table 2. Disinfection results on $20 \mathrm{~cm}$ FEP tubes with $0.9 \%$ saline solutions.

\begin{tabular}{|c|c|c|c|c|}
\hline \multirow[b]{2}{*}{ Samples } & \multirow{2}{*}{ 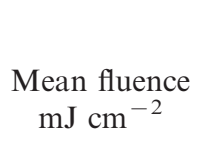 } & \multicolumn{3}{|c|}{ 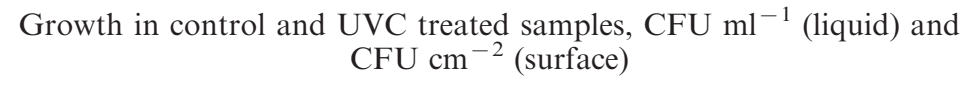 } \\
\hline & & Day 1 liquid & Day 3-4 liquid & Day 3-4 surface \\
\hline Control (2) & - & $9.2 \times 10^{3}(0)$ & $2.1 \times 10^{4}(0.2)$ & $8.5 \times 10^{3}(0.6)$ \\
\hline Saline control (2) & - & $2.0 \times 10^{3}(0.2)$ & $1.4 \times 10^{2}(1.4)$ & $0.7 \times 10^{2}(0.7)$ \\
\hline Control (3) $\Delta$ & - & $3.5 \times 10^{4}(0.08)$ & $3.3 \times 10^{3}(1.1)$ & $1.0 \times 10^{1}(0.3)$ \\
\hline Saline control (3) $\Delta$ & - & $1.0 \times 10^{3}(0.06)$ & $1.3 \times 10^{1}(1.2)$ & No growth \\
\hline UVC treated (4)\# & 0.22 & No growth & $5.1 \times 10^{2}(3.5)$ & $5.9 \times 10^{2}(5.4)$ \\
\hline UVC treated (3) $\Delta$ & 1.00 & No growth & No growth & No growth \\
\hline UVC treated (1) & 1.40 & No growth & No growth & No growth \\
\hline UVC treated (3) $\Delta$ & 1.40 & No growth & No growth & No growth \\
\hline UVC treated (3) $\Delta$ & 3.00 & No growth & No growth & No growth \\
\hline UVC treated (3) $\Delta$ & 5.60 & No growth & No growth & No growth \\
\hline UVC treated (1) & 5.62 & No growth & No growth & No growth \\
\hline
\end{tabular}

$20 \mathrm{~cm}$ FEP tube filled with $0.9 \%$ saline solution. The samples marked $\Delta$ represent a new series of measurements taken several months after the first series. The detection limits are $33 \mathrm{CFU} \mathrm{m}{ }^{-1}$ (day 1 ) and $3.3 \mathrm{CFU} \mathrm{ml}{ }^{-1}$ (days 3 and 4). The numbers in parentheses in column 1 are the number of replicates. Average CFU counts and SDs (numbers in parentheses) of the controls are shown in the last three columns. The UVC treated samples marked \# represent the average of doses ranging from 0.13 to $0.27 \mathrm{~mJ} \mathrm{~cm}^{-2}$ (treatment times from 30 to $60 \mathrm{~s}$ ). 


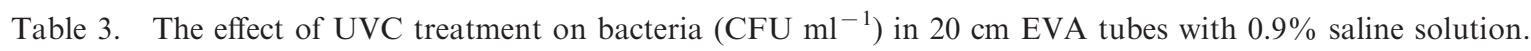

\begin{tabular}{|c|c|c|c|c|}
\hline \multirow[b]{2}{*}{ Samples } & \multirow{2}{*}{ 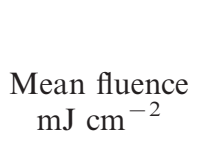 } & \multicolumn{3}{|c|}{$\begin{array}{l}\text { Growth in control and UVC treated samples, } \mathrm{CFU} \mathrm{ml} \mathrm{ml}^{-1} \text { (liquid) and } \\
\qquad \mathrm{CFU} \mathrm{\textrm {cm } ^ { - 2 }} \text { (surface) }\end{array}$} \\
\hline & & Day 1 liquid & Day 3-4 liquid & Day 3-4 surface \\
\hline Control (4) & - & $4.9 \times 10^{3}(3.5)$ & $3.5 \times 10^{4}(5.5)$ & $2.4 \times 10^{4}(2.4)$ \\
\hline Saline control (2) & - & $7.0 \times 10^{1}$ & No growth & 1 \\
\hline UVC treated (3) & 1.46 & No growth & Growth (\$) & $8.6 \times 10^{2}(\$)$ \\
\hline UVC treated (2) & 3.07 & No growth & No growth & No growth \\
\hline UVC treated (3) & 4.36 & No growth & $33(\$)$ & $8(\$)$ \\
\hline UVC treated (3) & 8.71 & No growth & No growth & No growth \\
\hline UVC treated (1) & 13.50 & No growth & No growth & No growth \\
\hline
\end{tabular}

The average fluence, each representing a different time setting, is given in the second column. The numbers in parentheses in column 1 are the number of replicates. The detection limits are $33 \mathrm{CFU} \mathrm{ml}{ }^{-1}$ (day 1) and $3.3 \mathrm{CFU} \mathrm{ml}{ }^{-1}$ (days 3 and 4). Average CFU counts and SDs (numbers in parentheses) of the controls are shown in the last three columns. Growth was observed in one of the replicates in items marked $\$$. The range of treatment times varied from 5 to 45 min.

Table 4. The effect of UVC treatment on bacteria in $20 \mathrm{~cm}$ EVA tube with a $P$. aeruginosa suspension $\left(c a 10^{4} \mathrm{CFU} \mathrm{ml}^{-1}\right)$.

\begin{tabular}{|c|c|c|c|c|}
\hline \multirow[b]{2}{*}{ Samples } & \multirow{2}{*}{ 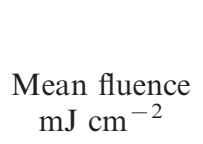 } & \multicolumn{3}{|c|}{$\begin{array}{l}\text { Growth in control and UVC treated samples, } \mathrm{CFU} \mathrm{ml} \mathrm{m}^{-1} \text { (liquid) and } \\
\qquad \mathrm{CFU} \mathrm{\textrm {cm } ^ { - 2 }} \text { (surface) }\end{array}$} \\
\hline & & Day 1 liquid & Day 3-4 liquid & Day 3-4 surface \\
\hline $\begin{array}{l}\text { Control (3) } \\
\text { UVC treated (3) }\end{array}$ & - & $\begin{array}{c}1.5 \times 10^{4}(0.7) \\
\text { No growth }\end{array}$ & $\begin{array}{l}8.4 \times 10^{3}(2.0) \\
7.0 \times 10^{1}(+)\end{array}$ & $\begin{array}{l}6.2 \times 10^{3}(0.6) \\
\text { No growth }\end{array}$ \\
\hline
\end{tabular}

The numbers in parentheses in column 1 are the number of replicates. The detection limits are $33 \mathrm{CFU} \mathrm{ml}^{-1}$ (day 1 ) and $3.3 \mathrm{CFU} \mathrm{ml}^{-1}$ (days 3 and 4). Average CFU counts and SDs (numbers in parentheses) of the controls are shown in the last three columns. No growth was observed in two of the samples, the item marked + , and the third sample was $2.2 \times 10^{2} \mathrm{CFU} \mathrm{ml}{ }^{-1}$. The treatment time was 75 min.

UVC treated $20 \mathrm{~cm}$ EVA tubes filled with $0.9 \%$ saline solutions, including those containing the $P$. aeruginosa solution, showed no growth on day 1 .

\section{Discussion}

The results obtained with the UVC disinfection set-up on the two types of tubes show common features but also some differences. No CFUs were found after day 1 , which was the case for both types of tube material and the three saline solutions used. Each of the saline control samples had high CFU counts on day 1. Based on this it was concluded that the UVC set-up was able to disinfect the contaminated tubes, including those FEP samples that were exposed for short periods of time (Table 1). The contamination level in the FEP and EVA tubes could be kept reasonably constant $\left(10^{3}-10^{4}\right.$ $\mathrm{CFU} \mathrm{m} \mathrm{m}^{-1}$ ) for a 3-4-day period (control samples in row 1, Table 4). All of the FEP control tubes, except the one in which the aseptic breach was replaced by a 10 or $20 \%$ saline control solution, were disinfected after 3-4 days. This seems to indicate that the high salt concentration was lethal for the planktonic bacteria present in the tubes. The results in Table $2(20 \mathrm{~cm}$ FEP with $0.9 \%$ saline solution) indicate that it is possible to determine a threshold fluence $\left(\sim 1.0 \mathrm{~mJ} \mathrm{~cm}{ }^{-2}\right)$ at which $100 \%$ disinfection is obtained. This dose corresponds to an exposure time of $5 \mathrm{~min}$ using the diode in these experiments. With new, more powerful diodes, this can be achieved in $1 \mathrm{~min}$, but shorter exposure times are difficult to administer in the clinic. Table 2 shows that the controls in the new set are substantially lower on days 3 and 4 compared to the first set. One possible reason for this is that the FEP tubes in the first set were re-used between experiments, thus providing the bacteria with anchor points formed by the brush used for removing bacteria attached to the surface. The results in Table 3 that show the EVA tubes with a $0.9 \%$ saline solution are surprising. The $0.9 \%$ saline control samples indicate that there were no viable cells on day 3 . A rather low contamination level was also observed on day 1 in the $0.9 \%$ saline control samples compared to that observed in the FEP tubes. Another striking feature is that small fluencies seem to be sufficient to obtain no growth despite the fact that it is known that the UVC light is poorly guided in the EVA tubes. The reason for this apparent contradictory behavior compared to that observed in the FEP tubes might be the result of the difference in surface structure of the two tube materials. Scanning electron microscope (SEM) images of the inner surfaces of new, unused FEP and EVA tubes were therefore taken 
(Figure 3). The two pictures are presented with the same magnification and are therefore comparable. The surface of the EVA tube was very smooth compared to that of the FEP tube, which had grooves from the extrusion process. It is believed that the short inoculation time combined with the smoothness of the inner surface of the EVA tubes meant that there were no, or few, anchor points for the bacteria, ie they were easily flushed away when the solution with the aseptic breach was replaced with the sterile $0.9 \%$ saline solution. If the bacteria were easily removed due to poor attachment, demonstrating the effectiveness of the UVC treatment on EVA tubes is difficult. To circumvent this problem, UVC exposure was thus used directly on the contaminated EVA tubes without replacing the solution with the aseptic breach. The results in Table 4 show that it is possible to disinfect the tubes ( 1 day of analysis). All of the other analyses carried out on days 3 and 4 showed no growth except one (liquid sample, see next section).

In comparison to what was observed earlier on established $P$. aeruginosa biofilm in FEP tubes, the following new observations are reported. In the early stage where the bacteria are present in the tubes for shorter time periods $(\sim 3 \mathrm{~h})$ smaller fluencies (shorter treatment times) are sufficient for disinfection. This was not the case for tubes with established biofilm. Substantially higher fluencies were necessary to kill bacteria that had been present for several days in the tubes. Fluencies $>0.29 \mathrm{~mJ} \mathrm{~cm}{ }^{-2}(1 \mathrm{~min}$ light exposure) are sufficient for this low level of contamination compared to $>16.6 \mathrm{~mJ} \mathrm{~cm} \mathrm{~cm}^{-2}$ (30 min) for biofilm. Keeping $20 \mathrm{~cm}$ Teflon and soft polymer tubes disinfected for 3-4 days is possible if the tubes are treated preventively with UVC light shortly after contamination. Furthermore, disinfection is also possible even though $0.9 \%$ saline solutions are used as the UVC light propagation liquids in the tubes during UVC exposure. It was also found that 10 and $20 \%$ saline solutions had

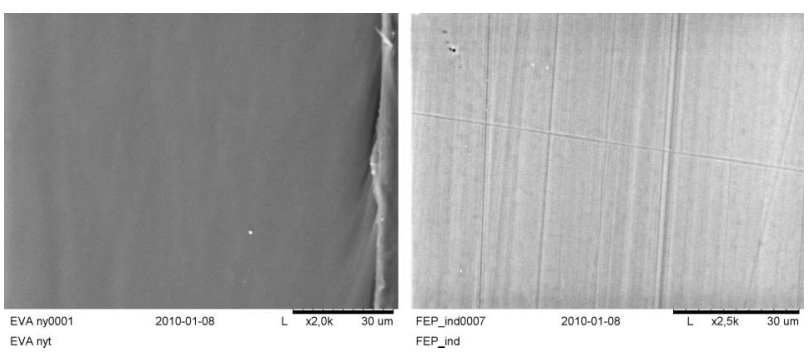

Figure 3. SEM images to illustrate the difference in roughness between the inner surfaces of new and unused polymer EVA (left) and FEP tubes (right). No grooves originating from the extrusion are visible on the surface of the EVA material. a strong bactericidal effect on these planktonic cells. Very few bacteria seem to survive after 3-4 days of exposure to these solutions.

\section{Sampling and diodes}

Two EVA samples ( $0.9 \%$ saline solution) showed CFU counts in unexpected cases (1.46 and $4.36 \mathrm{~mJ} \mathrm{~cm}^{-2}$ in Table 3 and 3-4-days in liquid in Table 4). In both sets of samples one of the three replicates gave bacterial counts. Opening the tubes on day 1 for liquid analysis may have caused them to become accidentally contaminated, thus subsequently accounting for the counts observed on days 3 and 4. A contaminated liquid film can be soaked into the space between the tube wall and the quartz stoppers used to seal the tubes on both ends. The UVC light is transmitted through the quartz stopper in the distal tube end during the UVC treatment. But due to its high refractive index (1.57) compared to the polymer, little or no UVC light is transmitted through the sides (confined inside the quartz by internal reflections), ie no disinfection will take place in the space between the stoppers and the tube wall. Both stoppers were removed for day 3 and day 4 sampling in order to empty the tubes and collect the liquid in the tube lumen, which simultaneously allowed for insertion of the pipette brush for sampling cells from the inner tube surface. Survivors from the space between the polymer wall and the stopper placed at the end opposite to the UVC diode were then mixed with samples drawn from the UVC-exposed tube lumen. When the aseptic breach solution was replaced by a high-concentration saline solution, the bacteria apparently died during days 3 and 4 . Typically, the salt in these high-concentrations solutions precipitates at the junctions between stopper and tube, making this area even more hostile to surviving bacteria. This is not the case when the $0.9 \%$ saline solution replaces the aseptic breach solution. Before long-term UVC disinfection experiments are carried out, the sampling procedure that apparently leads to contaminated tube lumens after opening and closing the tubes has to be solved. If UVC treated tubes filled with $0.9 \%$ saline solution are reopened and closed frequently, contamination of the lumen caused by the above-mentioned reasons is very likely.

The UVC diodes used in this study had highly limited output. In the experiments reported here, the output power was typically between 0.08 and $0.1 \mathrm{~mW}$. The development of more power-efficient diodes promises to translate into exceedingly short UVC treatment times. Diodes with a maximum output close to $10 \mathrm{~mW}$ at $270 \mathrm{~nm}$ are currently on the market. If these diodes are run with an output power of, for instance, $2 \mathrm{~mW}$, disinfection of a $20 \mathrm{~cm}$ high refractive 
index polymer tube (EVA, PUR, and silicone) can be achieved within a fraction of a minute if used preventatively. If UVC exposure is used on tubes/ catheters with established biofilm; disinfection might be possible with an exposure time of 10-20 min.

\section{Clinical features}

If a $0.9 \%$ saline solution is used as the light propagation medium, no chemical agents with possible and unknown side effects enter into the blood stream. The isotonic solution is normally part of the flushing procedure of the catheter lumen before heparin or another lock solution is injected. The UVC-based method allows a rapid kill within minutes of microorganisms entering and possibly colonizing the hub and catheter lumen. This is a short treatment time compared to reported preventative treatments with chemical agents such as citrate and taurolidincitra-lock solutions. In vitro tests of taurolidine-citrate solutions showed surviving bacteria $24 \mathrm{~h}$ after inoculation of the bacterial solutions, while no growth was observed $72 \mathrm{~h}$ after inoculation (Shah et al. 2002). Interestingly, high-concentration saline solutions $(10-20 \%)$ are reported here to have similar antibacterial properties against $P$. aeruginosa after $72 \mathrm{~h}$ (days 3 and 4). The quick kill obtained using the UVC method is especially significant if the catheter is accessed daily or several times in one day for drug or nutritional administration. The full preventative effect of the citrate, taurolidine-citrate and saline solutions can only be fully exploited if the chemical agent is in place for a longer period of time $(>24 \mathrm{~h})$. In addition, the short UVC exposure time needed for preventative treatment combined with the use of the isotonic solution for flush allow ordinary anticoagulants such as heparin to be injected and used as the lock solution immediately after UVC treatment. Another important feature is that the bacteria are killed starting with the top layer of the colonized catheter surface, where the most viable and motile microorganisms are expected to reside. The UVC method is not dependent on a completely clean inner surface. The light propagation still works if the liquid filling in the intra-luminal space is transparent, ie if contamination of the inner surface with biopolymers does not degrade the functionality of the method. Finally, it should be emphasized that UVC light kills all types of bacteria, Gram-positive as well as
Gram-negative. The tabulated doses necessary to obtain $99.9 \%$ kill for pathogenic bacteria relevant for catheter contamination are comparable. The 99.9\% kill doses for relevant bacteria (see for instance: http://www.uvp.com) are: S. aureus $6.6 \mathrm{~mJ}$ $\mathrm{cm}^{-2}, E$. coli $6.6 \mathrm{~mJ} \mathrm{~cm} \mathrm{c}^{-2}$, and $P$. aeruginosa $10.5 \mathrm{~mJ} \mathrm{~cm} \mathrm{~cm}^{-2}$. Work is in progress in which the UVC method is demonstrated to be effective against other inoculated organisms also.

\section{Acknowledgements}

This project has been supported by institutional grants and a grant from Region Zealand. The authors would like to thank E. Abdellahi from the Fuel Cells and Solid State Chemistry Department, DTU Risø, for providing the SEM images. Anne Grethe Holm Jensen and Tina Thane are acknowledged for their technical assistance and SP Medical A/S for providing the polymer tubes.

\section{References}

Ash SR. 2007. Fluid mechanics and clinical success of central venous catheters for dialysis: answers to simple but persisting problems. Semin Dialysis 20:237-256.

Bak J, Ladefoged SD, Tvede M, Begovic T, Gregersen A. 2010. Disinfection of Pseudomonas aeruginosa biofilm contaminated tube lumens with ultraviolet $\mathrm{C}$ light emitting diodes. Biofouling 26:31-38.

Diemer S, Meister J, Jung R, Klein S, Haisch M, Fuss W, Hering P. 1997. Liquid-core light guides for near-infrared applications. Appl Opt 36:9075-9082.

Donlan RM. 2001. Biofilms and device-associated infections. Emerg Infect Dis 7:277-281.

Donlan RM. 2008. Biofilms on central venous catheters: is eradication possible? In: Romeo $\mathrm{T}$, editor. Current topics in microbiology and immunology. Berlin: Springer Verlag. p. 133-161.

Menyhay SZ, Maki DG. 2006. Disinfection of needless catheter connectors and access ports with alcohol may not prevent microbial entry: the promise of a novel antiseptic-barrier cap. Infect Cont Hosp Ep 27:23-27.

O'Grady PN. 2002. Applying the science to the prevention of catheter-related infections. J Crit Care 17:114-121.

Raad II, Hanna HA. 2002. Intravascular catheter-related infections. Arch Intern Med 162:871-878.

Shah CB, Mittelman MW, Costerton JW, Parenteau S, Pelak M, Arsenault R, Mermel LA. 2002. Antimicrobial activity of a novel catheter lock solution. Antimicrob Agents Chemother 46:1674-1679.

TexLoc. 2005. Refractive index of polymers: extensive tabulation for visible wavelengths [Internet]. Available from: http://www.texloc.com.

Weast RC. 1977-1978. CRC handbook of chemistry and physics. 58th ed. Boca Raton (FL): CRC Press. p. D252D253. 